\title{
Charting global position and vision of stakeholders towards sustainable bioenergy
}

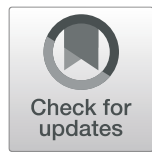

T. Mai-Moulin ${ }^{1 *}$, U. R. Fritsche ${ }^{2}$ and M. Junginger ${ }^{1}$

\begin{abstract}
Background: Stakeholder's position of bioenergy sustainability is important for the deployment and contribution of bioenergy to sustainable development. Existing publications are usually limited to specific geographical contexts and focuses. This paper aims more broadly to examine the position and vision of a wider range of stakeholder groups towards bioenergy and its development at a global level.

Method: The applied methodology includes six steps: (1) identification of stakeholders as belonging to one of seven groups; (2) describing the role of each group in relation to bioenergy; (3) data collection via an online questionnaire, roundtable dialogues and interviews to examine their stated awareness and opinions of bioenergy development, driver and barriers to such development; (4) data analysis; (5) comparison of interests and influence as a basis for expressing position and vision; and (6) recommendations for gaining support for sustainable bioenergy development.

Results: The stakeholders state awareness of bioenergy development and have in general a positive view of the sector. They also inform that the general public is less aware of and not sufficiently involved in bioenergy development. Internet and social media are the most consulted sources of information but least trusted, while scientific information is most trusted but least used. Agricultural residues, energy crops cultivated on marginal or degraded land and forestry residues are widely accepted as feedstocks for bioenergy production, whereas use of agricultural land is viewed critically. The stakeholders generally support bioenergy development when jointly agreed sustainability requirements are met.
\end{abstract}

Conclusions: The stakeholders acknowledge the important role of effectively disseminating scientific information as an influencing factor on the position towards bioenergy. They also find that enhancing support for the bioenergy sector relies on mandatory sustainability requirements covering social, economic and environmental aspects, applied to all types of biomass regardless of end use. Some also emphasise that all relevant sectors should work on market conditions to create a level playing field and that this is crucial to change stakeholders' position to gain more social acceptance of bioenergy. Transparency in demonstrating compliance with sustainability criteria is also an expected pre-condition to enhance support for bioenergy (and ultimately the bioeconomy) in the long term.

Keywords: Bioenergy, Sustainability, Bioenergy supply chains, Stakeholders' position, Stakeholders' vision, Sustainability criteria, Sustainability requirements, Certification

\footnotetext{
* Correspondence: t.p.t.mai-moulin@uu.nl

${ }^{1}$ Copernicus Institute of Sustainable Development, University of Utrecht,

Vening Meinesz Building A, Princetonlaan 8a, 3508 TA Utrecht, The

Netherlands

Full list of author information is available at the end of the article
}

(c) The Author(s). 2019 Open Access This article is distributed under the terms of the Creative Commons Attribution 4.0 International License (http://creativecommons.org/licenses/by/4.0/), which permits unrestricted use, distribution, and reproduction in any medium, provided you give appropriate credit to the original author(s) and the source, provide a link to the Creative Commons license, and indicate if changes were made. 


\section{Background}

Bioenergy has an important role in the current and future energy landscape [1]. Many European Union (EU) member states and other countries have recognised the role of bioenergy in increasing the share of renewable energy and reducing greenhouse gas (GHG) emissions, in helping countries to become less dependent on fossil fuels, and in making a significant contribution to the bioeconomy [1-7]. In the EU, energy from biomass and the renewable share of waste contributes to almost two thirds of the primary combined renewable energy production today, and the absolute amount is expected to further increase slightly until $2030[8,9]$. Other large economies have also emphasised the role of bioenergy on their policy agendas. In 2017, modern bioenergy accounted for about $50 \%$ of total global renewable energy consumption, and bioenergy is expected to be the largest source of growth in renewable energy consumption over the period 2018-2023, with an expected growth of 30\% in this period [7]. The EU 2020 and 2030 renewable energy targets as well as country-level policies and energy efficiency will increase the use of bioenergy in the continent [6]. Also, in its latest 2018 report, the Intergovernmental Panel on Climate Change (IPCC) highlights that bioenergy use is substantial in $1.5^{\circ} \mathrm{C}$-consistent pathways due to its multiple roles in decarbonising energy use with or without a carbon capture and storage combination [10].

The potential role of bioenergy in the transition to a sustainable low-carbon energy system is widely acknowledged by stakeholders in many countries and regions. However, the extent to which bioenergy should be deployed and under what conditions, what feedstocks should be used and what end uses should be stimulated in the short-, medium- and long-term are seen differently by diverse stakeholder groups. According to various regional case studies, many stakeholders indicate recognition of the positive role of bioenergy in the current and future energy landscapes and support bioenergy development if certain conditions are met, but many other stakeholders hold negative views on bioenergy if certain conditions are not satisfied [8, 11-13]. However, those studies mainly focus on specific supply chains in regions or countries in Europe, the United States (US), and Asia [11, 13, 14]. Many of them focus on specific aspects such as the challenges to governance of bioenergy sustainability [12], social acceptance of energy issues [15] or on a limited range of stakeholder groups, mainly the general public or consumers $[13,16]$. Those studies have answered questions related to relatively isolated issues such as feedstock preferences for bioenergy, multi-level governance and impacts of governance on bioenergy production and trade, awareness about bioenergy, and attributes driving opinions about bioenergy. According to Radics et al. [13], most studies are carried out either an online survey or interviews to assess stakeholders' views. Only $4 \%$ of the studies combined both communication methods.

This study is accomplished as part of the IEA Bioenergy project "Measuring, governing and gaining support for sustainable bioenergy supply chains". Two of the project objectives are to (a) understand the position and underlying motivations of diverse stakeholders related to their role in bioenergy development and (b) inform dialogue and discussion to avoid misconceptions as well as provide neutral and comprehensive knowledge on the bioenergy sector. To achieve this objective, three regional case studies are conducted to assess the views and position of stakeholders relevant for three bioenergy value chains at regional and national levels: biogas in Germany [17] and woody biomass for energy and agricultural biomass for biofuels in Canada [18] and the US [19]. These studies were co-funded by various national programmes and, therefore, also used slightly different approaches. However, there is a lack of studies that analyse the position and vision of stakeholders towards bioenergy (1) at a global level and which also (2) deploy multiple methods at the same time to assess, verify and consolidate the results. Such a comprehensive study is important to provide a clearer picture of how bioenergy is viewed, also from a global perspective. As the international trade of bioenergy products is increasing, it becomes more critical to have information on position of stakeholder groups from local to global levels, which can guide the bioenergy industry to develop sustainably. This is a pre-condition to the legitimacy of the bioenergyrelated activities.

To fulfil the objectives, the stakeholders are divided into eight different stakeholder groups and data are collected through various consultation channels including an online questionnaire, roundtable dialogues and indepth interviews. The roundtable dialogues allow stakeholders to discuss and validate results of the online questionnaire and the interviews, thus elucidating the position and vision expressed by supranational stakeholders. The study also gives an improved understanding of the position and underlying motivations of diverse stakeholders relative to their role in bioenergy. Finally, the study indicates how the position of stakeholders and their institutions may affect bioenergy policies and the sector development in the future.

\section{Methodology}

A variety of methodologies has been developed and applied to identify, map and understand stakeholders' position and vision as well as their influence on the bioenergy sector. For example, an applicable framework for the assessment, design, implementation and communication of 
quality stakeholder engagement was developed to identify stakeholder groups, their position and perception to understand their expectations about governance, policies and strategies concerning bioenergy [20]. Other frameworks were developed for specific case studies with the aim to analyse stakeholders' viewpoints and social acceptance of biomass projects $[12,15,21]$. Taking into account various approaches used in previous studies including their shortcomings, a method was developed for this study, which focused on classification of stakeholder groups and assessment of stakeholders' position and vision by using a number of communication channels. We defined perception is the ability of stakeholders to understand an issue and position of stakeholders as their ability to perceive and give opinions about that issue. In this study, perception was briefly investigated based on their responses to general information of the bioenergy sector whilst position was measured as the level to which a stakeholder agrees with a statement from strong disagreement to strong agreement level. Vision was defined as the ability to think and plan for the future based on the expertise and experience. Once stakeholders provided statements how they view bioenergy in the short, medium and long term, their vision was observed. The method included six steps (Fig. 1).

The first step involved the identification and classification of eight stakeholder groups and screening of their roles. Stakeholder groups were classified as either being directly involved in the bioenergy supply chains or not; the former included biomass producers, biomass users for bioenergy and biomass users for other purposes (such as stakeholders of the biomaterials and biochemical industries). Outside of the supply chains, stakeholder groups included the general public (consumers, local communities), academia and consulting (research institutions, consultancies), non-governmental organisations (NGOs) and policy-makers. There was also a supranational stakeholder group which is considered active and interested in bioenergy on an international level, rather than on a specific case level. They show an understanding and awareness of bioenergy.

The second step was to define the different roles of the stakeholder groups. Biomass producers are actors who supply feedstocks and are involved in the cultivation, harvesting and collection, storage, and logistics of biomass. Biomass users for bioenergy and for other purposes are stakeholders involved in the bioeconomy sectors or in the supply chains of biomass for various purposes: logistics, pre-treatment and conversion of feedstocks to products of the bioeconomy. The other stakeholders outside the supply chains have some interests in bioenergy and bear some levels of influence on bioenergy development. The supranational stakeholders are the stakeholders with more influence on bioenergy development. As this paper focuses on the potential role of bioenergy on a global level in the medium to long term, most of consulted supranational stakeholders have a clear vision on the role of bioenergy in the long-term timeframe. However, it was not always evident what the main perception and drivers for this vision are. Therefore, communication with selected supranational stakeholders was particularly important to analyse how they vision the bioenergy sector and to explore how bioenergy could move forward sustainably.

The third step was to communicate with stakeholders via questionnaires, interviews, and dialogues to understand their viewpoints and influence on the bioenergy sector. The communication approaches (see Table 1) are explained in the "Online questionnaire", "Roundtable dialogues with invited stakeholders" and "Interviews with supranational stakeholders" sections. For the roundtable dialogues with selected stakeholders and interviews with the supranational stakeholders, the communication outcomes were documented and sent to those stakeholders for their review and approval. The communication results published in this paper have been approved by all stakeholders.

The fourth step was to capture information about stakeholders' position, awareness and interest inside and

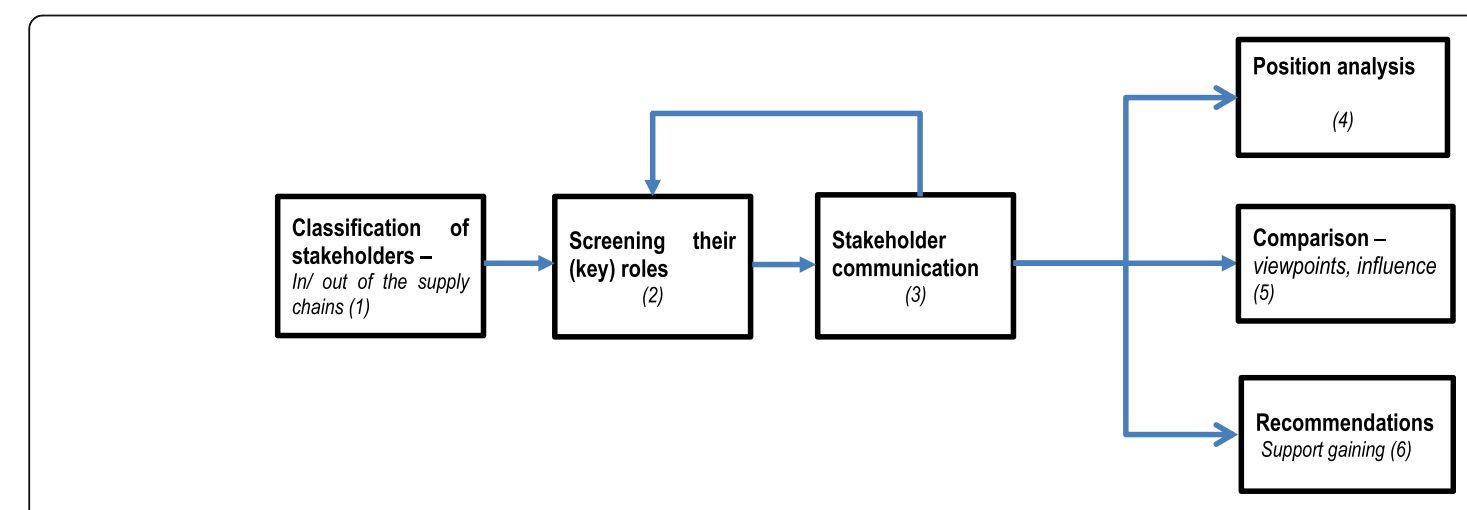

Fig. 1 Method to identify stakeholders' position, perception and vision on bioenergy 
Table 1 Contributions from different communication approaches

\begin{tabular}{llll}
\hline Issues addressed & $\begin{array}{l}\text { Online } \\
\text { survey }\end{array}$ & $\begin{array}{l}\text { Roundtable } \\
\text { dialogues }\end{array}$ & Interview \\
\hline Position of stakeholders & $\mathrm{X}$ & $\mathrm{X}$ & $\mathrm{X}$ \\
$\begin{array}{l}\text { Perception towards } \\
\text { bioenergy }\end{array}$ & $\mathrm{X}$ & $\mathrm{X}$ & $\mathrm{X}$ \\
$\begin{array}{l}\text { Vision on bioenergy } \\
\begin{array}{l}\text { Influence on bioenergy } \\
\text { development }\end{array}\end{array}$ & $\mathrm{X}$ (partly) & $\mathrm{X}$ \\
\hline
\end{tabular}

outside the bioenergy supply chains, then interpreted why certain stakeholders have more influence than others (and in what contexts).

The fifth step was to compare stakeholders' viewpoints and vision of different bioenergy value chains and to the bioenergy sector. This step also aimed to understand and predict the level of interests and influence of stakeholders on bioenergy development in order to provide future recommendations following the approach of Reed et al. (2009) [22]. The figure of interest and influence was drawn to map stakeholder interests and influence with inputs from the dialogues and interviews with supranational stakeholders.

The final step provided discussion and proposed recommendations for how the bioenergy sector can gain (further) support from stakeholders for sustainable bioenergy development and value chain management.

\section{Online questionnaire}

The questionnaire was developed with the aim to receive a brief poll of perception and vision of seven stakeholder groups towards the bioenergy sector. The questionnaire covered various aspects of bioenergy development, established through discussion with bioenergy consultants and scientists who participated in this project. The aspects included stakeholders' awareness of bioenergy development; conditions under which to support bioenergy; suitable feedstocks to be used for bioenergy production; drivers, barriers and challenges for bioenergy development; and how to gain (further support) for bioenergy sustainability. The questionnaire was reviewed and approved by the project Advisory Board. There were several questions which included five levels of agreement: strong agreement, agreement, neutral viewpoint, disagreement and strong disagreement (stakeholders could also provide their own opinions) (Additional file 1).

The questionnaire was disseminated via the project participants to their networks, to several websites of IEA Bioenergy and its members. In addition, the questionnaire was also announced at a number of events and conferences in which project members participated. Once the questionnaire was completed, an analysis of position, viewpoints and vision of the stakeholder groups was carried out. The analysis included the average ranking of agreement and acceptance levels by the stakeholder groups. Also, the standard deviation of the respondents' answers was quantified. A low standard deviation indicates that answers tend to be close to the average ranking, while a high standard deviation indicates that responses are spread out over a wider range of values.

\section{Roundtable dialogues with invited stakeholders}

The roundtable dialogues were designed to consult a number of stakeholders having interests and expertise on bioenergy to receive feedback on the questionnaire results, and to reflect their vision for future pathways and strategies for sustainable bioenergy development. The stakeholders mostly had a position which may have influence to and clear vision on bioenergy development. They jointly answered four questions:

- Which results of the online questionnaire are in line with their expectations?

- Which results of the online questionnaire are unanticipated? Are their own areas of disagreement with other stakeholders the same as those emerging from the questionnaire?

- Are there other main areas of disagreement?

- What are possible areas of agreement (or where agreement may be achieved fairly easily)?

\section{Interviews with supranational stakeholders}

A number of organisations that relate to or have influence on bioenergy development were identified. This investigation of relevant organisations was also done through communication with or recommendations from other stakeholder groups. Supranational stakeholders representing selected organisations approached included experts and policy-makers from the European Commission and Parliament; United Nations (UN) organisations; World Bank and regional development banks; international NGOs (e.g. Greenpeace, IUCN, WWF); bio-based industry (e.g. DSM, Unilever) and their EU and global associations (e.g. Bioenergy EUROPE, WBA); fossil fuels-based industries (e.g. BP, Shell); EU and international forest owner associations, selected forest-related industries; biomass sustainability certification bodies (e.g. Programme for Endorsement of Forest Certification Schemes (RSB), International Sustainability \& Carbon Certification (ISCC)), including forest certification bodies (e.g. Forest Stewardship Council (FSC), Programme for Endorsement of Forest Certification Schemes (PEFC)); international agricultural businesses; and land owner associations.

The supranational stakeholders were identified based on their published information and relevant works linked directly or indirectly to the bioenergy supply 
chains. Their communication, presentations and publications on biomass and bioenergy issues were also reviewed. The invitations were delivered to the supranational stakeholders and once agreed to participate in this study, they firstly answered the questionnaire then responded to eight questions via an interview. The interviews were designed to identify the vision of their institutions' involvements in bioenergy or their influence on the development of the bioenergy sector. The additional questions include:

a. Public involvements in bioenergy projects: What would be the recommendations and to what level of involvements?

b. Involvement of supranational stakeholder's organisation in bioenergy. What projects and or programme have done related to biofuels/bioenergy sustainability?

c. Bioenergy end use: What are the most important end uses of bioenergy?

d. Perspectives: What are the perspectives on the bioenergy market, trade, willingness to pay?

e. Sector policies: What would be the most important policies for bioenergy development?

f. Recommendations: Under what conditions could the bioenergy actors gain (further) supports from external stakeholder groups?

g. EU policies on bioenergy: The EU is a good example of bioenergy development and sustainability compliance. The EU has ambitious targets for bioenergy including biofuels, heat and electricity sectors. How can the targets be met?

h. How do you view the sector in the short, medium and long terms?

Their responses to the questionnaire were reviewed and discussed in the interviews to ensure clear answers and explanations of all the identified issues. Once the questionnaire and interviews were completed, an assessment of the position, viewpoints and vision of the supranational stakeholders was carried out. This assessment investigated how these stakeholders viewed the roles of various bioenergy supply chains. Also, the assessment revealed whether the current actions of the supranational stakeholders match their long-term vision. In case their answers were inconsistent, further communication and investigation was also carried out for detailed clarifications. The assessment aimed to identify to what extent there was a coherent vision among different supranational stakeholders. The assessment aimed to answer whether the supranational stakeholders exerted an influence on the bioenergy supply chains and on development of the bioenergy sector in the medium and long term.

\section{Results}

\section{Online questionnaire}

Stakeholder participation

The online questionnaire received contributions from more than 200 participants, with 199 of these being complete for further data processing. The contributions came from all the seven defined stakeholder groups including contributions of the supranational stakeholders (see Fig. 2).

The questionnaire received most contributions from the academia and consulting group (35\%), with the second largest group being NGOs (17\%), policy-makers (15\%) and biomass users for energy (12\%). Participation rates from other groups were lower than $6-8 \%$. This constituted a fair number of stakeholder groups and it is thus expected that the results reflect well the interests and involvement of stakeholder groups in the bioenergy sector.

\section{Awareness}

In general, the respondents declared that they were aware of bioenergy development and had a rather positive view of the sector, with the most positive views from the biomass users for energy, NGOs and academia and consulting groups. Together with policy-makers, these three groups showed the highest level of self-expressed awareness of the sector's activities. The biomass users for bioenergy expressed the highest level of awareness of bioenergy development. The respondents generally indicated that the general public is too little aware of bioenergy, and this group should be more informed and involved in the development of the sector. In designing bioenergy policy, the respondents did not agree that the general public should have more influence than scientific evidence. The general public themselves did not consider their voice more important than scientific facts (see Fig. 3).

The standard deviation showed that within the stakeholder groups, the answers were varying compared with the average level of agreement.

\section{Feedstocks used for bioenergy}

The results concerning feedstocks used for bioenergy production (see Fig. 4) showed a high level of acceptance (average 70\%) for the use of agricultural residues (harvesting and processing crop residues), energy crops cultivated on marginal or degraded land as well as forestry residues (from conventional harvest operations, processing, urban wood and low-value wood). This acceptance originated mostly from biomass producers, biomass users for energy, NGOs, policymakers, academia and consulting groups. Many stakeholders indicated their choices for those agricultural and forest feedstocks for the reason that these 


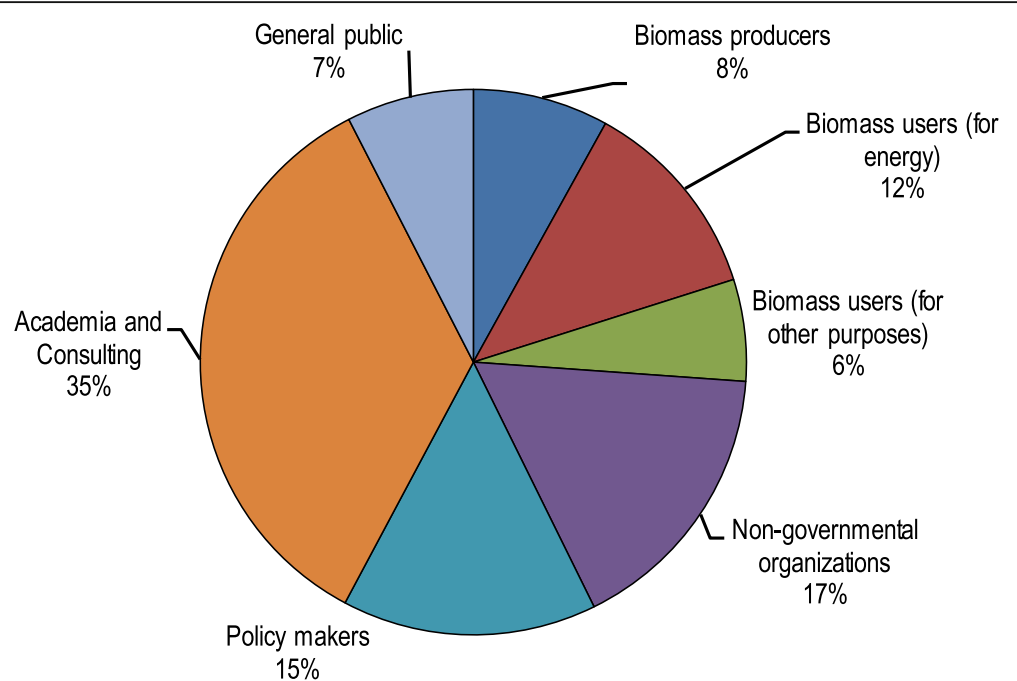

Fig. 2 Relative distribution participants of the seven stakeholder groups in the online questionnaire, with contributions of the supranational stakeholders among the answers as belonging to some of the other groups

\section{AGREEMENT LEVEL:}

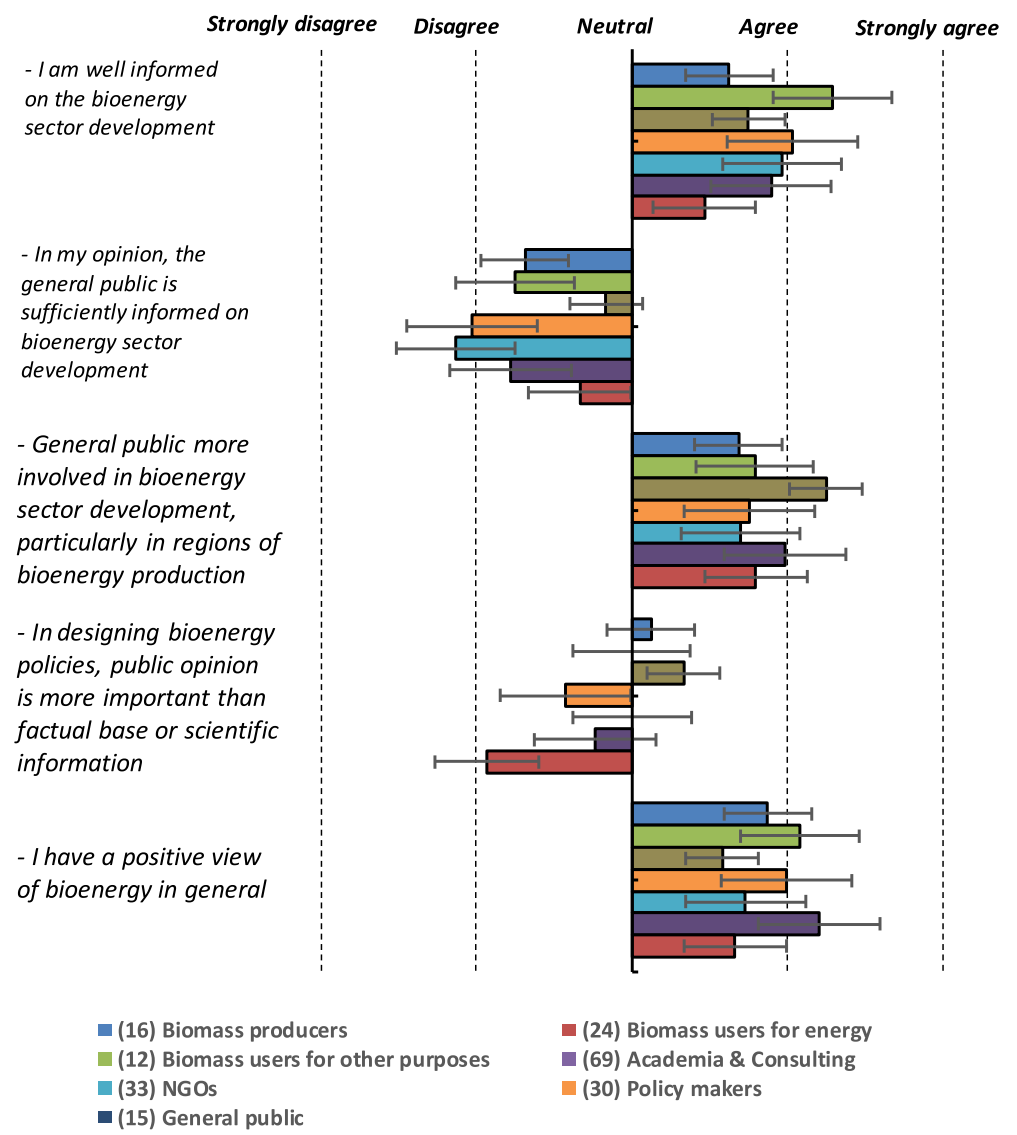

Fig. 3 Stakeholders rating of their own awareness and general view on bioenergy sector development. Bars show that answers varied most among stakeholders within the groups Biomass Users for Energy, Academia \& Consulting and NGOs 


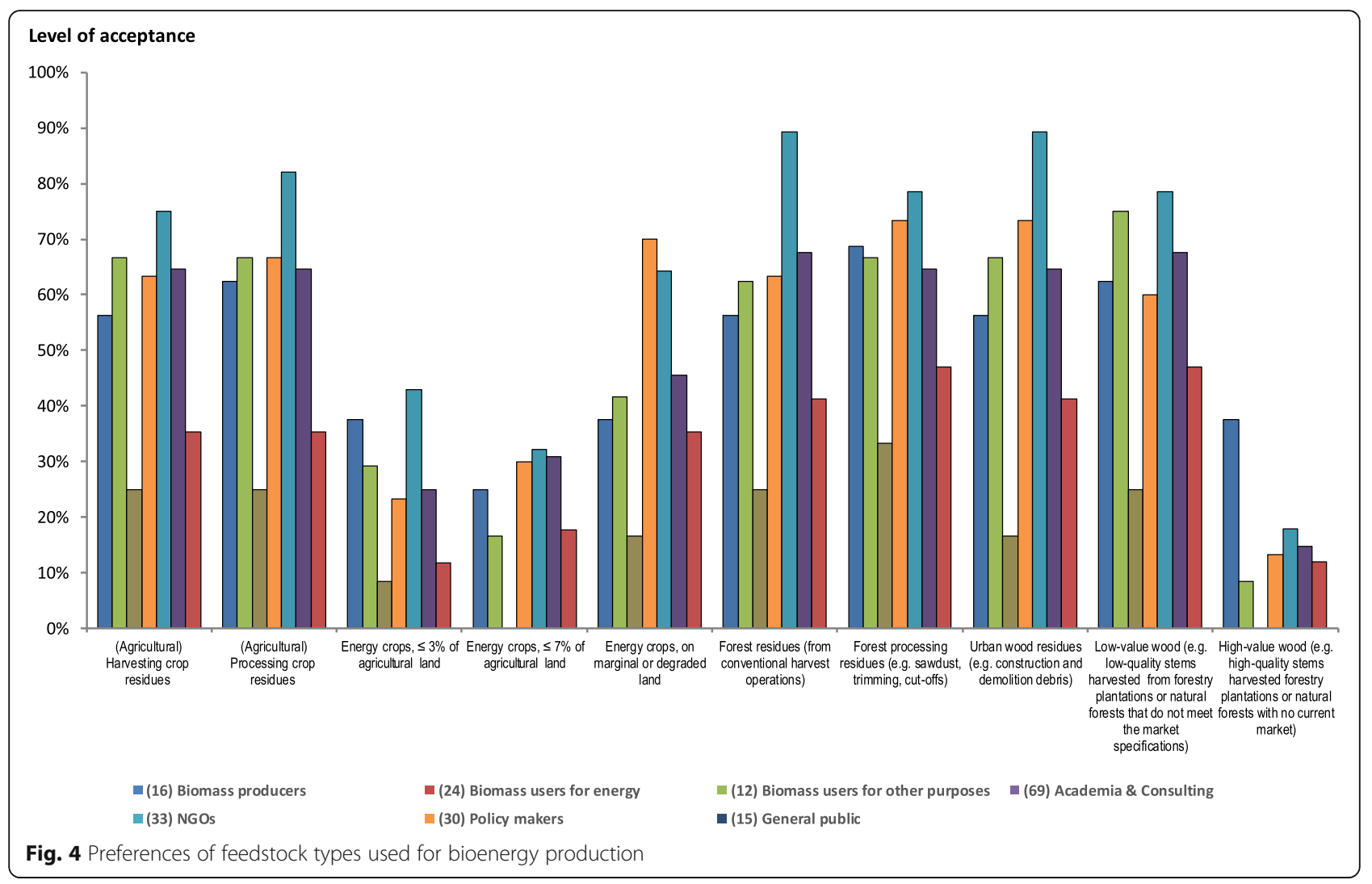

biomass feedstocks bear a low sustainability risk if used for bioenergy production. Biomass users (for other purposes than bioenergy) indicated a low acceptance of various feedstock types used for bioenergy production.

Most energy crops grown on a certain percentage (e.g. $\leq 7 \%, \leq 3 \%$ of the land area) or the use of high-value wood harvested from plantations and natural forests (even those with no current market) for bioenergy received little support from any stakeholder group.

\section{Information about the bioenergy sector}

The stakeholders were asked what sources of information they consulted about bioenergy and what sources of information they trusted most. Results show that internet sources, social media, television, local events and traditional newspapers provided a high percentage of the information (more than $70 \%$ stated these are their main sources, see Fig. 5). Meanwhile, publications from academia and consulting-although they did not contribute significantly to the popular dissemination of

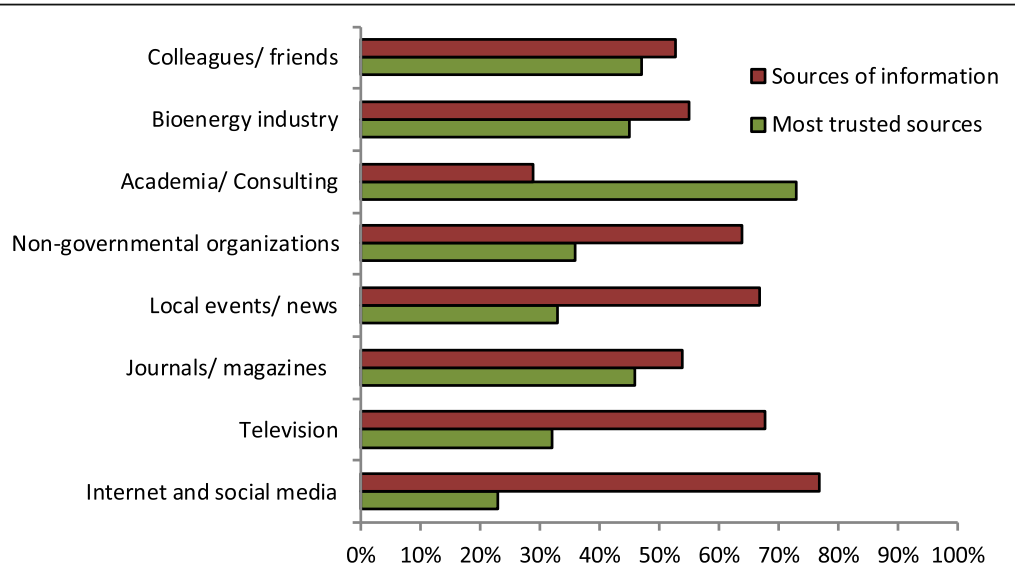

Fig. 5 The types of consulted sources and most trusted information sources about the bioenergy sector 


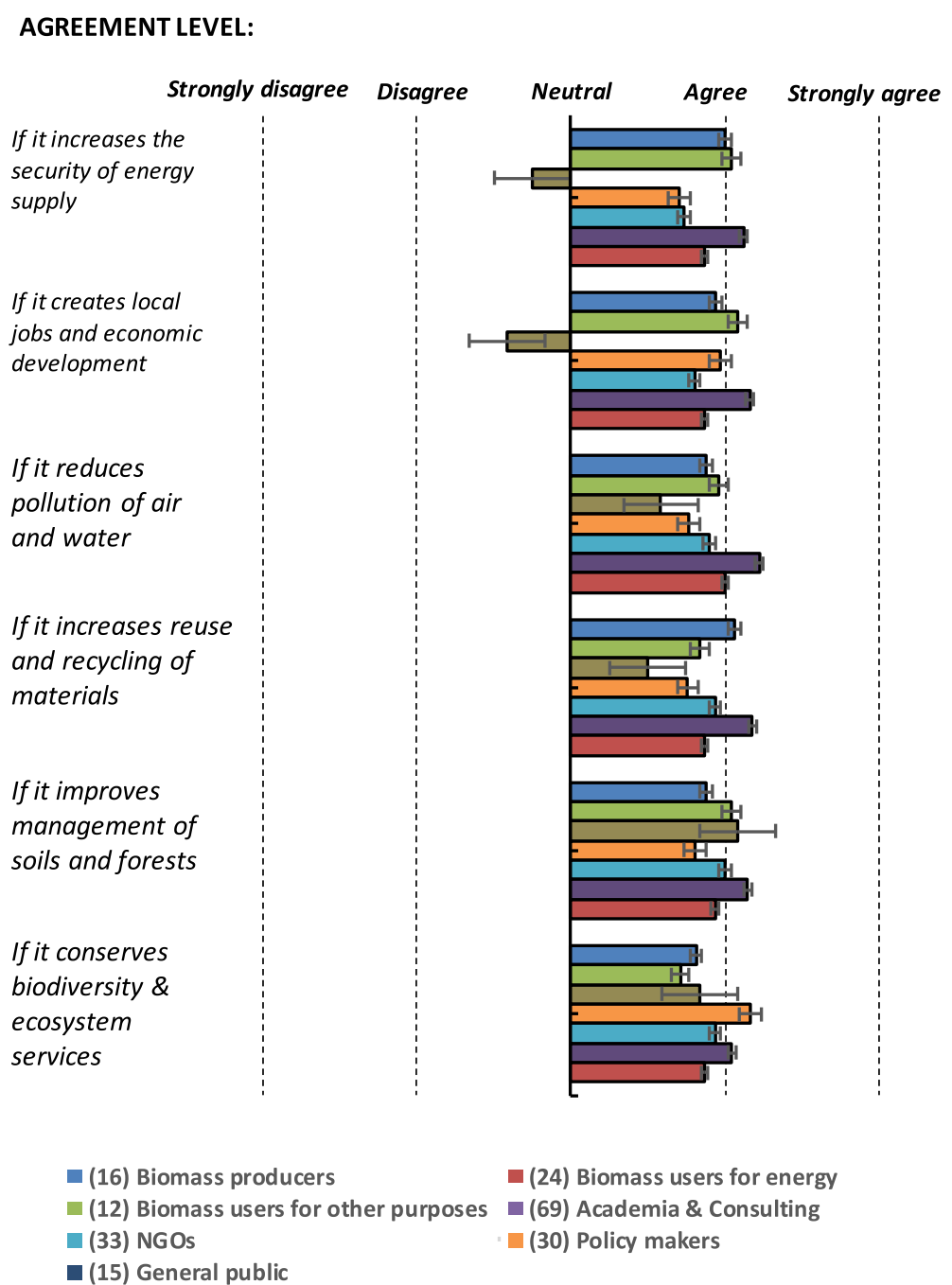

Fig. 6 Stakeholders rating of reasons for their support of bioenergy development. Bars show a low variation of answers within each group, with highest varying answer for the group Biomass users for other purposes

information-were considered the most credible information sources. In addition, some stakeholders expressed that they gained knowledge about bioenergy through their own experience and network.

\section{Support or disapproval of bioenergy}

The stakeholders were asked whether they support bioenergy development based on 6 stated arguments: increase of energy supply security, local job creation and economic development, reduction of air and water pollution, increase of reuse and recycling of materials, improvement of soil and forest management, and conservation of biodiversity and ecosystem services. Most of the stakeholder groups agreed that these aspects were all important (see Fig. 6). The highest agreement came from the academic and consulting group.

The biomass users for other purposes disagreed with the aspects (a) increase of energy supply security, and (b) local job creation and economic development. In addition, they provided additional comments that other sectors such as pulp and paper, and construction traditionally create more jobs and stimulate more economic development than the bioenergy sector.

The stakeholders were also asked if they would disapprove of further bioenergy development if it leads to a number of negative impacts. All stakeholders agreed on three issues: over-exploitation of forest or deforestation, land rights conflicts and especially negligible or no reduction of GHG emissions as reasons to not support bioenergy development (see Fig. 7). Furthermore, all stakeholder groups took a neutral position on competition with material uses.

For other impacts, the results were more varied. Potential indirect land use change (iLUC) was considered a serious reason by the general public to disapprove bioenergy, whereas all other stakeholder groups were close to a neutral view on this issue. Regarding the lack of 


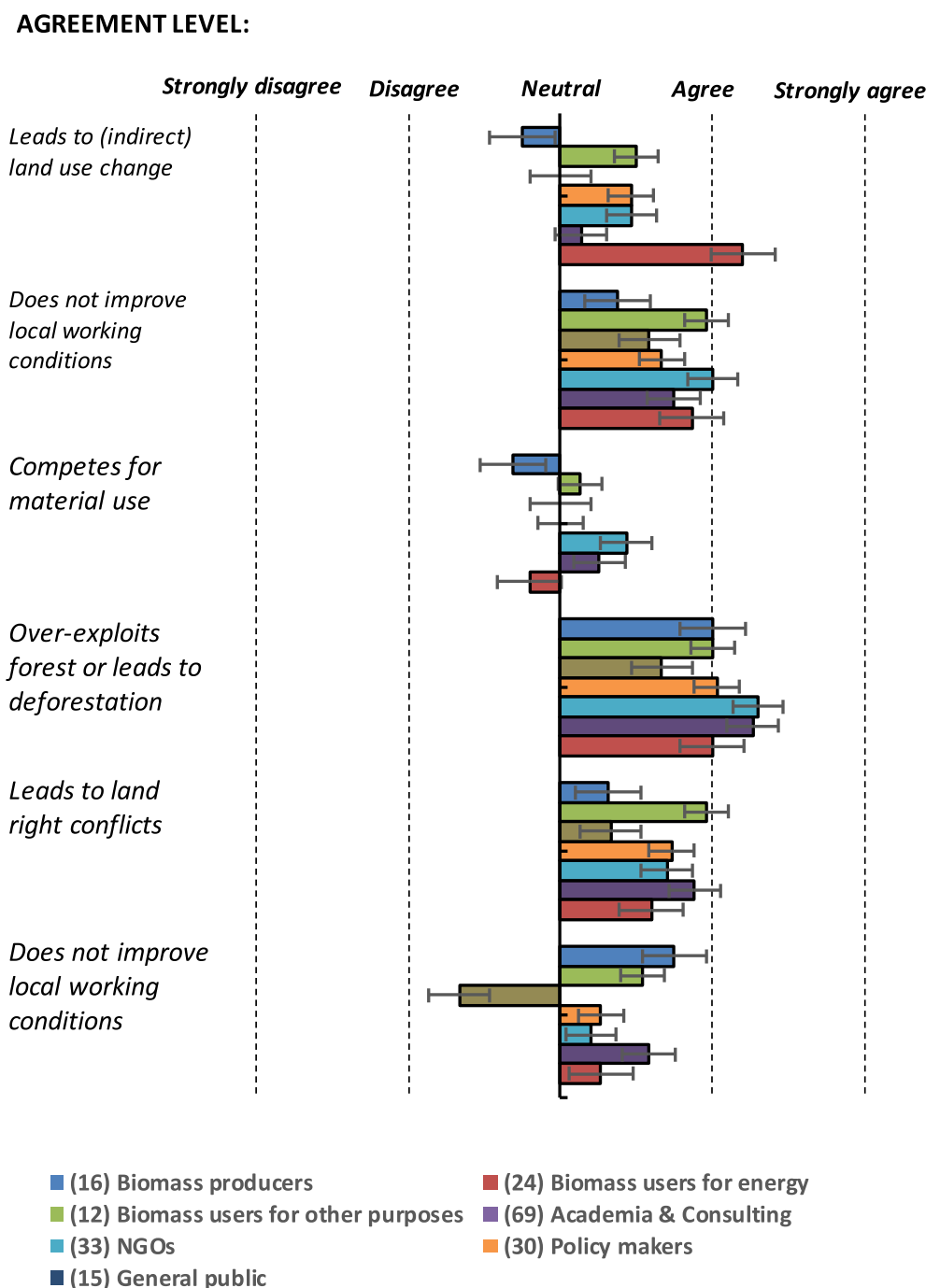

Fig. 7 Stakeholders rating of reasons to disapprove of bioenergy development. Bars show the same level of variation of the answers within all groups

improvement of local working conditions, the biomass users for other purposes, did not consider this an issue, whereas all other groups saw this as a minor reason to disapprove of the bioenergy sector.

\section{Barriers and challenges for the bioenergy sector}

Most of the stakeholder groups considered the lack of economic stimulation and market incentives as well as unresolved sustainability issues (resulting in policy and market uncertainty) as the two main barriers to bioenergy development. The lack of scientific information for better informing policy-makers and the general public was also viewed as a barrier but deemed less important than the two aforementioned aspects (see Fig. 8).

A key challenge for the bioenergy sector, agreed upon by most stakeholders, is the lack of general social acceptance. Undesired environmental impacts which cannot be avoided or no contribution to economic growth were not considered serious issues according to all stakeholder groups (see Fig. 9).

\section{Drivers and barriers to bioenergy development}

The stakeholder groups were also asked if they agreed with the five presented drivers of bioenergy development: reduction of GHG emissions, reduction of environmental impacts, creating profitable businesses based on biomass, development of a circular economy, energy security and security of energy supply. In principle, all stakeholder groups agreed that these factors are drivers but indicated their highest agreement with the reduction of GHG emissions (see Fig. 10).

The stakeholder groups were also asked how the bioenergy sector could gain or enhance support. Most stakeholders agreed with the position that one of the most promising ways 


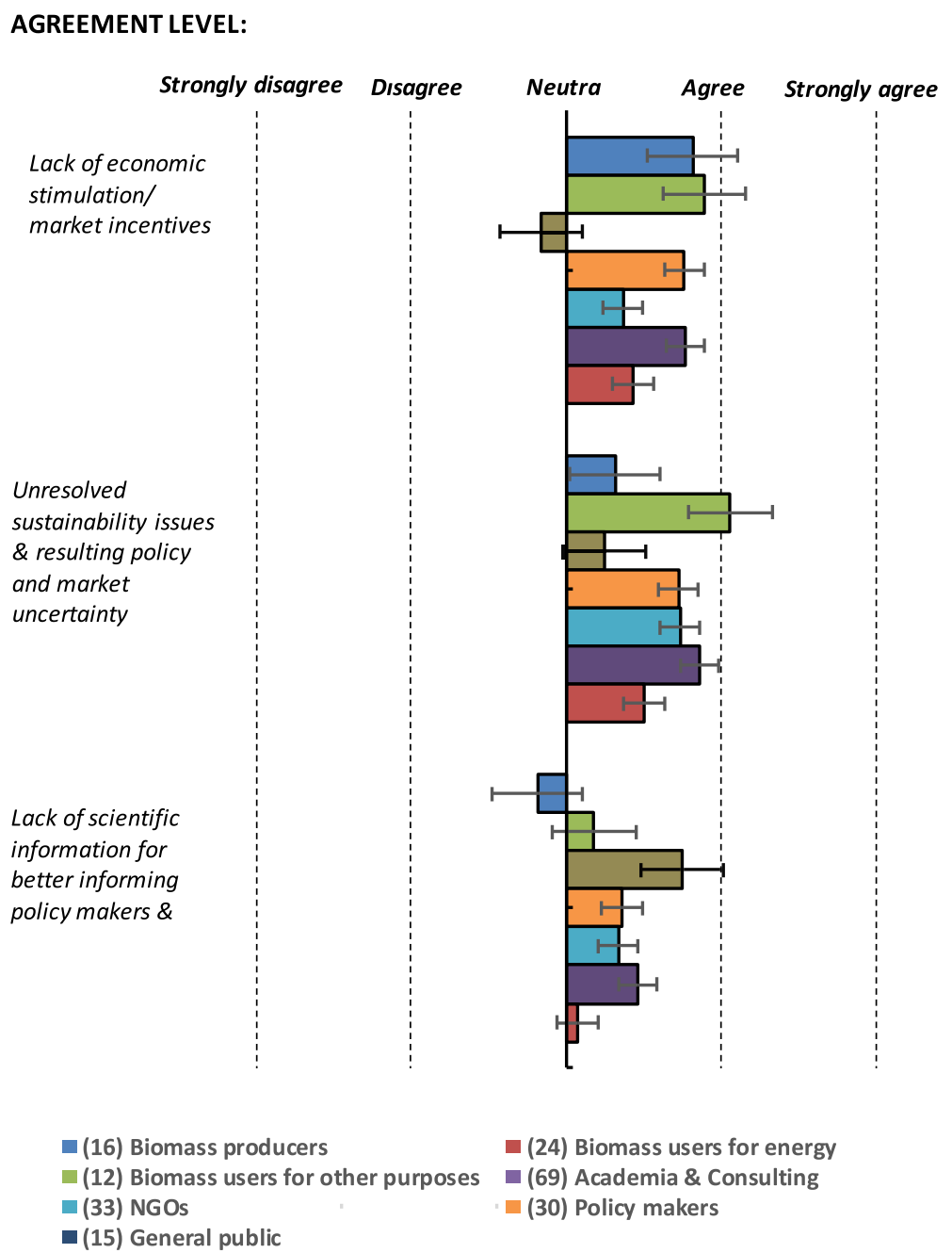

Fig. 8 Stakeholders rating of barriers to bioenergy development. Bars show answers varied most within the groups Biomass producers, Biomass users for energy and Biomass users for other purposes

is the introduction of binding sustainability requirements for all types of biomass feedstocks, whether used for bioenergy but (remarkably) or other end uses. Especially, the bioenergy end-users showed the highest agreement. Another wellsupported position was to increasingly base bioenergy policies on scientific information. The stakeholders did not completely consider that current certification schemes are transparent and effective indicating that further improvements are needed (see Fig. 11).

\section{Roundtable dialogues}

The questionnaire results were discussed in two roundtable dialogues. ${ }^{1}$ The two events engaged many

\footnotetext{
${ }^{1}$ International conference "Governing sustainability of bioenergy, biomaterial and bioproduct supply chains from forest and agricultural landscapes" in April 2018 and the IEA Bioenergy side event "Sustainability and governance of bioenergy supply chains" at the 26th European Biomass Conference and Exhibition in May 2018.
}

stakeholders. A total of fifteen stakeholders participated, representing NGOs (Danish Society for Nature Conservation (DN), World Wide Fund for Nature (WWF) Europe, Global Biomass Partnership (GBEP), Confederation of European Forest Owners (CEFP)), two independent consultants, biomass users for energy (Hofor, Enviva, Drax), a certification scheme (Roundtable on Sustainable Biomaterials (RSB)), industry associations (Danish Energy, Brazilian Sugarcane Industry Association (Unica)), an union (United Federation of Danish Workers (3F)), and intergovernmental organisations (Food and Agriculture Organisation of the United Nations (FAO), International Renewable Energy Agency (IRENA)).

\section{Anticipated results in line with participants' experience}

Overall, more than half of the participants stated that the positive view of the stakeholder groups was in line with 


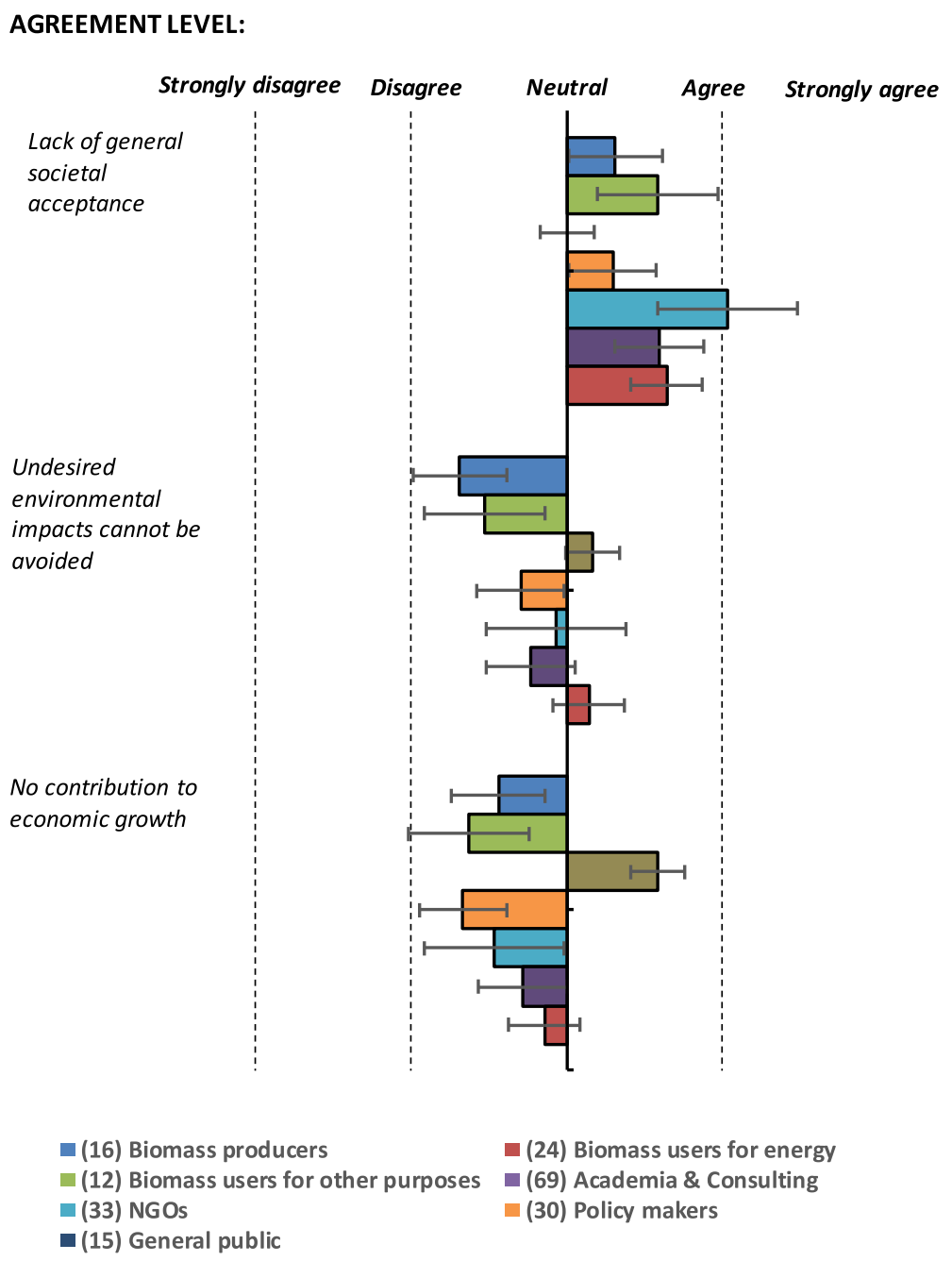

Fig. 9 Challenges for bioenergy development

their experience, and they commented that bioenergy receiving the support of many stakeholder groups was a good signal for the bioenergy sector. They agreed with most of the questionnaire respondents that GHG emissions reduction was the main driver of bioenergy development. Also, the view that the general public was not aware of bioenergy activities with more efforts needs to be made to inform the general public was confirmed. The concerns of negative impacts of bioenergy expressed by the general public, and a lack of social acceptance of bioenergy were also recognised by the dialogue participants.

The participants confirmed that they understood why the biomass users for other purposes often gave answers differently than other groups as this is consistent with this group's interests. The participants commented that this group uses biomass feedstocks that are similar to those used for bioenergy production and that feedstock competition was likely an aspect that made this group reluctant to support the development of the bioenergy sector. Most participants commented that the bioenergy sector should take the concerns of this group into account.

The participants also supported the view expressed in the questionnaire concerning drivers, barriers and challenges for bioenergy development, showing that social and economic aspects should be taken more into consideration as sustainability dimensions. The participants noted that sustainable forest management (SFM) and some social and economic aspects have been implemented in some EU member states. However, clear definitions for these criteria need to be agreed upon at a global level.

\section{Unanticipated results in line with participants' experience}

Half of the workshop participants commented that NGO's positive view of bioenergy was surprising and they recommended contacting additional environmental NGOs to receive more opinions. This was consequently done. It did not change general impression as presented in the "Online questionnaire" section, even though this 


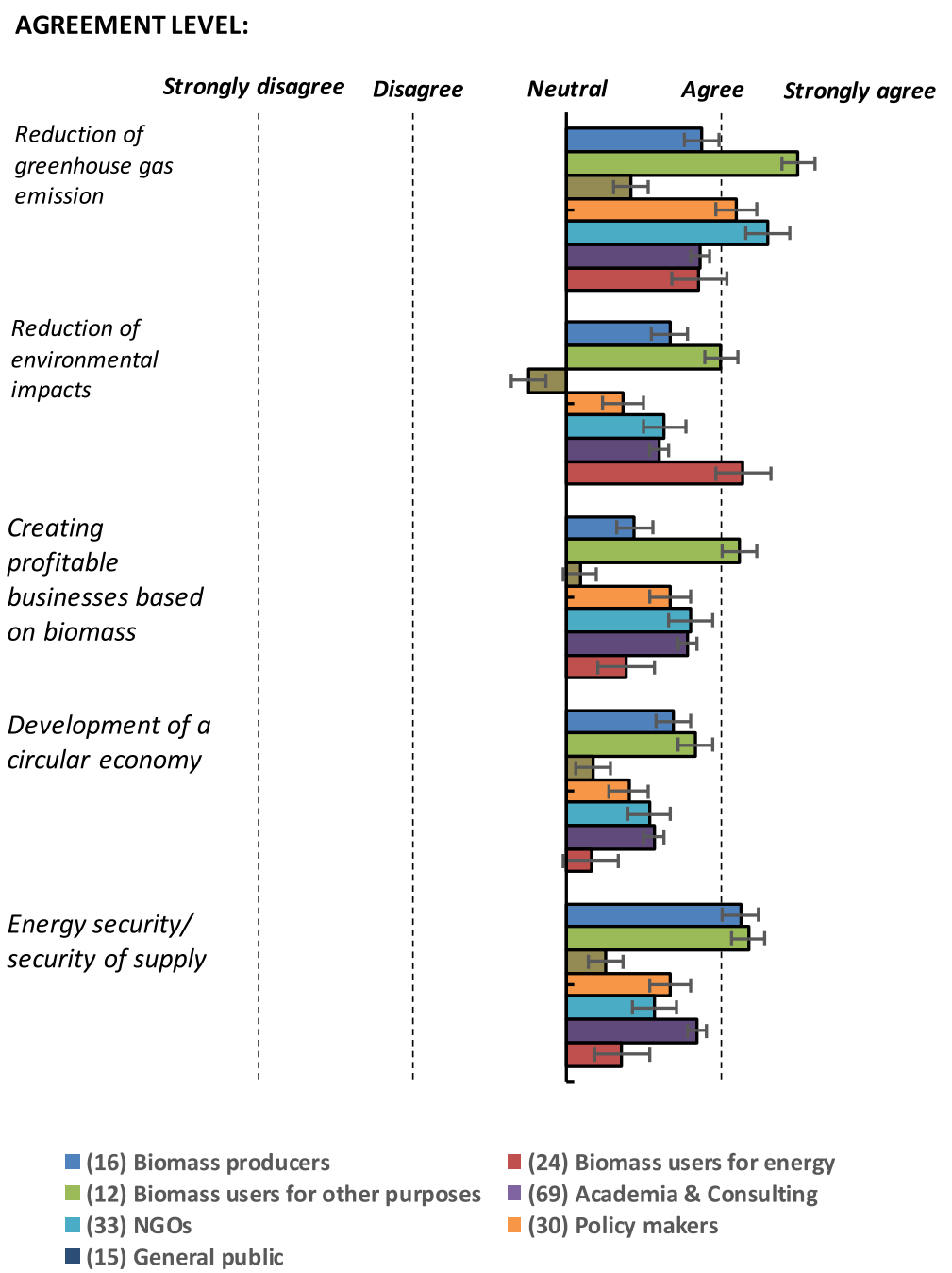

Fig. 10 Stakeholders' rating of the importance of drivers of bioenergy development. Bars show a low variation of answers among all groups

group was somewhat less positive compared with the view presented in these events. This may be because many participating NGOs did not represent only environmental groups, but also included bioenergy industry associations, forest and farmer associations, who generally support bioenergy development.

Some participants stated the social and economic benefits of the bioenergy sector should be highlighted as a driver. Regarding information sharing, the participants indicated their disagreement that information from the bioenergy sector is not especially trusted, while that from the academia and consulting is more trusted. Other participants noted that there was a lack of understanding about bioenergy, and the contributions of bioenergy to the bioeconomy. The participants were negatively surprised that mandatory sustainability requirements for bioenergy as well as bioenergy policy based on scientific information did not receive (even) higher levels of support than shown in Fig. 11. They agreed, however, that improvements are still needed for the certifications schemes which are already deemed transparent and effective. According to them, more improvements would still be needed for the certification schemes, for them to be more inclusive and more comprehensively measure sustainability indicators for bioenergy.

Regarding feedstock use, several participants expressed concerns regarding the low support for energy crops, except on marginal and degraded lands, as these feedstocks are important for expanding bioenergy. They found that bioenergy crops would be acceptable in addition to other biomass feedstocks, if sustainability compliance were established and compliance demonstrated. They also highlighted that with improved yields for food crops, there would be more land available for bioenergy crops and that could have an important role in decarbonising the transport sector. In addition, information sharing and generally sustainable biomass were emphasised as important to gain or enhance support for bioenergy. 
AGREEMENT LEVEL:

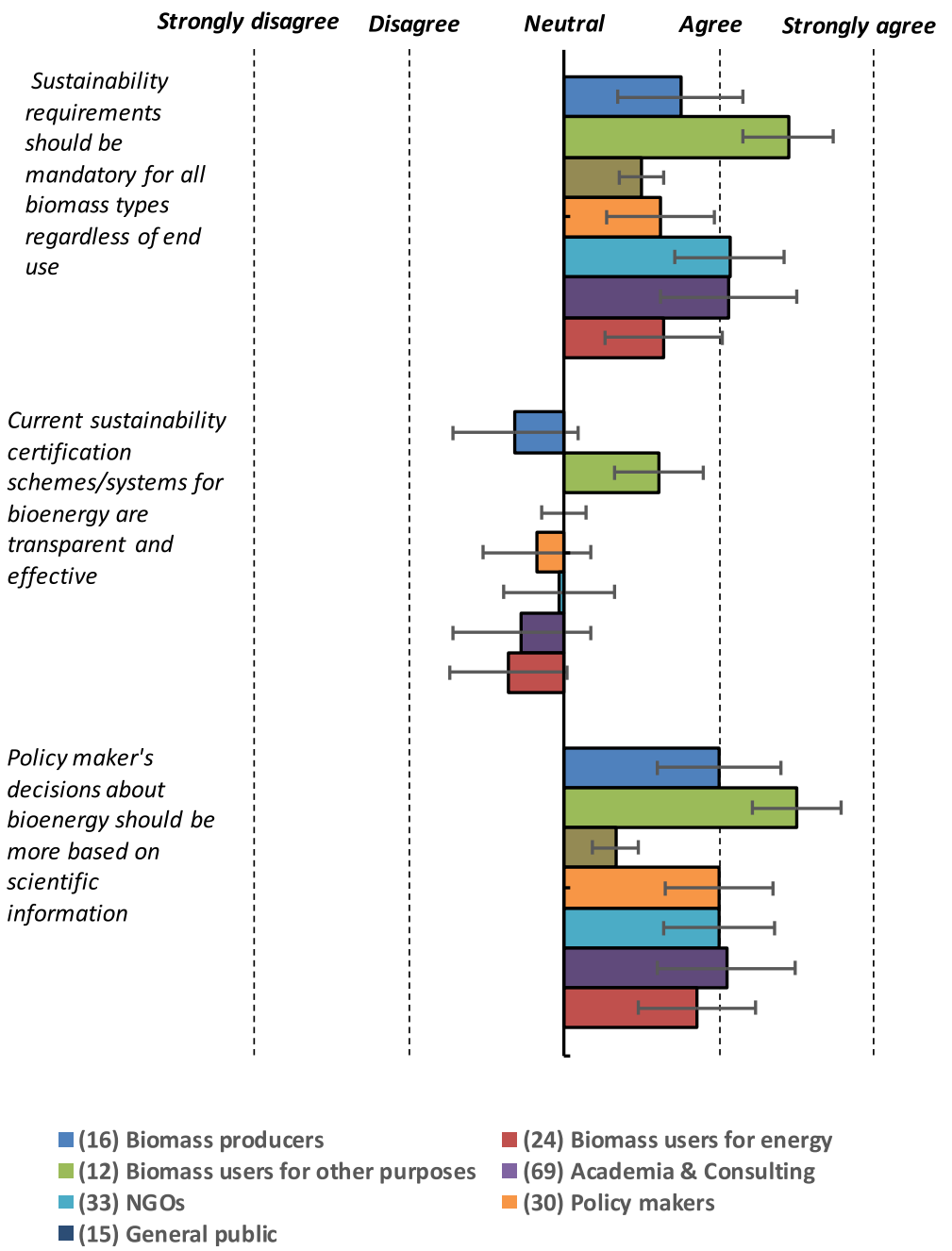

Fig. 11 Stakeholders' rating of conditions to gain (further) support for bioenergy development. Bars show highly varying answers among stakeholders in most groups

They also emphasised that market mechanism are important for creating a fair distribution of biomass feedstocks for all relevant sectors.

\section{Other areas of disagreement with the questionnaire results}

The participants also identified the lack of scientific information for better informing policy-makers and the general public, as a barrier to bioenergy development, highlighting that such information needs to be carefully presented to external stakeholders. It was also seen as a challenge that scientific papers present conflicting results on bioenergy. This was considered to confuse stakeholders, particularly those actors who have influence on sector development. Other participants mentioned that bioenergy debates would be needed to identify which results are credible. Also, information was seen as needed on how bioenergy can make contributions to economic growth.
Regarding sustainability safeguards for bioenergy, many stakeholders agreed that implementing sustainability requirements for bioenergy at a global level is inappropriate; sustainability verification should be carried out at a local scale to assure accurate outcomes, in particular in relation to sustainable land management.

\section{Other areas of agreement with the questionnaire results}

Some participants believed that bioenergy has an important role in the future energy system, but also that the sector would need to take the Paris Climate Agreement into account. In order to keep climate change globally below $1.5^{\circ} \mathrm{C}$, GHG emissions need to be reduced dramatically through afforestation and reforestation. They also considered that a percentage of forest biomass could be made available for bioenergy, if necessary to agree which forest types that are suitable for sustainable 
biomass harvesting, particularly for tropical forests and rainforests. They also expressed the importance of avoiding forest over-exploitation, for example by implementation of low LUC and iLUC measures. The general public should also receive information on land use effects of biomass cultivation and harvesting, including the positive as well as negative effects of bioenergy, if evidence of such effects can be provided. They saw that energy crops as well as forest waste and residues are sensitive topics in terms of gaining support and considered that such support might depend on the expected usage and volumes. They suggested as a solution that agreements be reached on feedstock use, biomass cascading and biomass applications in various sectors.

Many participants emphasised that vision on bioenergy should be clear and more explicit to guide the sector to a proper development. They expressed that policies missing a long-term perspective are barriers to investment. Transparency and communication with other related sectors and external stakeholders were deemed helpful for bioenergy to strengthen its position, as was enhanced collaborations between the bioenergy sector and the scientific community for communicating their scientific results to NGOs and policy-makers. They said that bioenergy policies should then be based on facts, but also that information delivered to policy-makers must comprehensible for this non-expert group. Similarly, information about bioenergy needs to be adequately simple for the general public to understand the development and its benefits and impacts.

Several participants noted that the social components, such as income and job creation, and local development should be included as voluntary sustainability indicators in the sourcing regions of biomass feedstocks as showing compliance is costly and time-consuming and does not involve critical risks. If economic operators comply with such voluntary requirements, they may receive premium for bioenergy production (in some regions) and or social acceptance, increase market share (for sustainable bioenergy). These components are particularly important in the developing countries where there is often a lack of stringent regulation or their enforcement or lack of compliance with local rights and laws. Social, economic and environmental dimensions and compliance with laws or guidelines were considered helpful, for the bioenergy sector to be supported by other stakeholder groups.

\section{Interviews with supranational stakeholders}

Out of 30 invitations sent, 11 supranational stakeholders agreed to answer the questions. Several supranational stakeholders did not respond to the interview invitation including a number of US policy-makers, fossil-fuel based industry, one development bank and international economics organisations.

The following supranational stakeholders clearly stated that the answers given represented the views of their organisation: DSM (bio-based/chemical industry), Transport \& Environment (NGO), Greenpeace (NGO), RSB (certification scheme), RVO (Policy department, Netherlands Enterprise Agency), Bioenergy Europe (bioenergy industry organisation) and UNEP (UN organisation). Other supranational stakeholders, although representing institutions with a significant influence on bioenergy development, presented their answers as personal opinions. They explained that their organisation did not have broad activities covering all aspects identified in the questionnaire and in the interview questions. Those stakeholders included representatives from Hofor (bioenergy industry), Rainforest Alliance (NGO), World Bank (development bank) and FAO (intergovernmental organisation).

The opinions and answers of the supranational stakeholders to the eight questions identified in the "Interviews with supranational stakeholders" section are presented below. Most of the institutions to which the supranational stakeholders (or the supranational stakeholders themselves) have published reports and position papers on bioenergy [23-28]. Many supranational stakeholders have also participated in dialogues, discussion and projects on bioenergy and provided references to further support their opinions and perception [29,30]. In the interviews, they elaborated further on their vision for bioenergy.

\section{Public involvements in bioenergy projects}

The questionnaire results showed that most of the supranational stakeholders agreed strongly that the general public is not sufficiently aware of bioenergy development and the general public should be more involved in bioenergy project implementation. In the interviews, they stated that public concerns and debates need to be recognised, for example when considering bioenergy effects combating climate change, food security, sustainable forest management, or impacts on human rights and land rights.

Three supranational stakeholders noted that raising awareness through bioenergy campaigns should be prioritised, with the general public involved in provisioning of local information and report environmental impacts of bioenergy projects. For sustainable value chains, local communities could contribute to feedstock sourcing and supply, and sustainability compliance. It was also suggested that these communities could also be consulted for public policy development, even if a potential risk of bad decision-making as seen as an aspect that also needed to be considered. 
Most supranational stakeholders recognised that bioenergy has various forms produced by various technologies delivering different end uses, thus making it difficult to derive a clear picture of what bioenergy truly is. According to them, it is important to develop communication strategies to inform the public of actual bioenergy activities and future plans.

\section{Awareness and participation on bioenergy sustainability}

Eight of the 11 supranational stakeholders indicated a clear vision on bioenergy, and the majority stated that they have knowledge on bioenergy markets, policies and sustainability criteria and certification. Many had been involved in developing and discussing legislation for bioenergy in Europe and its member states, as well as in implementing and managing sustainability compliance for bioenergy. They were familiar with and involved in projects of feedstock mobilisation with the participation of companies, landowners, forest communities and partner organisations.

However, the role of bioenergy and its contributions to climate change mitigation was still in question by three supranational stakeholders. Those stakeholders commented that bioenergy needs to be sustainable if the sector should continue to grow. In addition, some supranational stakeholders had concerns that, the actual GHG emissions reduction under certain conditions was not as high initially assumed. They had a positive impression of that the EU revised Renewable Energy Directive 2018 (RED II) defining binding sustainability for the whole bioenergy sector to enhance sustainability compliance. The RED II was seen by some stakeholders to have weak requirements for GHG emission reductions. However, they believed the RED II will be helpful to harmonise sustainability criteria among member states and will respond to the sustainability concerns of the general public. They concluded that similar sustainability criteria should be adopted at the global level.

\section{Prioritisation of bioenergy end uses}

All the supranational stakeholders mentioned that modern bioenergy is particularly important in the transport sector. Eight supranational stakeholders also considered end uses in the heat and electricity sectors to be important. Three supranational stakeholders emphasised the role of bioenergy in aviation, and for biomaterials and maritime uses. However, most of them also highlighted that the end uses should rely on regional policies and emphasised a consideration of environmental and climate protection, and possible environmental costs. Four participants recommended further considering the cascading principle, using feedstocks of high quality for biofuel or biomaterial production, while waste and residues should be processed for heat and electricity.
Six supranational stakeholders mentioned that local contexts of bioenergy production play a role for its development. Biofuels are becoming popular in the US, South America and the EU, but biofuels consumption is small outside of these regions. For further development, it was seen as important to carefully assess sustainability, particularly land-related issues.

\section{Perspectives on bioenergy market, trade and willingness to pay}

Seven of the 11 supranational stakeholders stated that sustainability certification can help in establishing bioenergy trade. It can also increase willingness to pay for bioenergy, even if it might be difficult to document in developing countries due to a lack of effective governance and institutional frameworks. They saw it not only as a barrier for trading, but also as an opportunity to mobilise more scattered feedstocks in other regions. Development of sustainable value chains would create extended markets for bioenergy, ultimately with customers willing to pay for sustainable bioenergy uses.

Four supranational stakeholders also emphasised that market price, market transparency and cost effectiveness play important roles in bioenergy sector development. Bioenergy markets in many countries are mainly policy driven, with bioenergy depending on subsidies, carbon prices, etc. Other renewable energies (solar panels, wind power) are becoming cheaper and thus more competitive and would likely gain markets. But four others stated that the bioenergy sector is gradually moving forward thanks to its contributions to secure energy supplies and stabilisation of electricity grids with increasing but intermittent shares of solar and wind power.

\section{Important policies for the sector development}

Seven supranational stakeholders emphasised that concrete policies for bioenergy were not yet on the political agenda in many countries. Policies should be well designed and implemented to support sustainable growth. Also, policies should help incentivise bioenergy to successfully compete in markets with fossil fuels. The following points were considered important policies:

- Sustainable sourcing and fair competition: six supranational stakeholders highlighted these issues as important to address in policies for bioenergy. The use of biomass feedstocks should be fair and avoids competition with other bioeconomy sectors using the same feedstocks. Policies guiding the bioenergy sector to develop sustainably and enhanced collaborations with related sectors are considered key for sustainable long-term development. 
- Carbon accounting and energy price: six supranational stakeholders indicated that sustainable bioenergy produced from biomass in principle emits much lower levels of GHGs than fossil fuels. A joint climate and energy framework as well as energy policies taking into account environmental and social costs in the final energy prices should be developed. This will ensure a fairer competition among energy carriers and it would be important for the sector to grow further and receive further political support.

- Sustainability requirements: according to five supranational stakeholders, comprehensive sustainability criteria are needed, both for the production of the biomass and conversion to end uses. The EU binding sustainability criteria are a good example to demonstrate how sustainability compliance can be extended to other regions of the world, through sustainability certification of voluntary schemes such as the FSC or the ISCC. Concrete sustainability criteria, definitions and sustainability monitoring for bioenergy need to be established for global issues, for example, iLUC. However, consensus on measures at a global level to has not been reached. The supranational stakeholders noted that ensuring no land competition for food could help gain more support for bioenergy. In addition, SFM criteria need to be more broadly applied so that biomass collection does not have adverse impacts on biodiversity and ecosystem conservation.

- Decarbonisation and aviation: five supranational stakeholders argued that policies on decarbonising the transport sector would be very important. Also, a global Emissions Trading System (ETS) and related efficiency requirements would stimulate the bioenergy sector to develop further. Four supranational stakeholders saw potentials for bioenergy in aviation to be further expanded, and thus policies for aviation could be a significant driver.

\section{Recommendations for the bioenergy sector to gain support} Recommendations from the supranational stakeholders for the bioenergy sector to gain support are as follows:

- Sustainability compliance and transparency: for six supranational stakeholders, these aspects are important for the bioenergy sector to receive support. Proper implementation of certification would ultimately lead to more confidence in bioenergy investment. Providing information for sustainability reporting and verification of bioenergy development should therefore be on the agenda of bioenergy stakeholders.

- Bioeconomy: three supranational stakeholders mentioned that a level playing field for all bio-based sectors, including bioenergy, is important.

- Information and communication: According to six supranational stakeholders an open attitude towards sustainability is the key to opening the door to the bioenergy future, involving several sectors in finding mutual solutions (e.g. bioenergy is not the main issue of the forest sector in which deforestation is a big general challenge to overcome). Recognition of different opinions would be needed as well as a sustainability path that is truly meaningful for society as a whole. Bioenergy should thus lead to real reduction of GHG emissions and contributes to energy security and to local development in the short and long term.

- Promotion and dissemination of bioenergy benefits: five supranational stakeholders emphasised that communication of opportunities and concerns regarding the development of bioenergy to policymakers is important. The bioenergy actors should disseminate knowledge on the use of biomass for energy from scientific, technological, economic, social, and legal angles to external stakeholders. Also, it was considered important to advocate the abolition of any technical or trade barriers which hamper the development of an open bioenergy market. It was finally recommended that bioenergy actors demonstrate a clear and consistent approach of using efficient resources.

\section{Bioenergy in the EU and target achievement}

Four supranational stakeholders mentioned that EU policies have stimulated higher targets for renewable energy share and liquid biofuels in several countries and that these targets will likely play a more significant role in the future. The development in the EU can provide perspectives for bioenergy development in other world regions. Consistent and long-term legislations would help to ensure long-term investments, while comprehensive sustainability criteria, robust certification systems, and implementation of best practices are also crucial for receiving more support.

\section{Bioenergy in the short, medium and long term}

Most supranational stakeholders highlighted that the development of bioenergy would depend on various factors. The perception, position of policy-makers and the policy framework are very important to pace the future development of bioenergy. Enhanced dissemination of the scientific findings, and under which circumstances bioenergy can make a positive contribution to 
sustainable development are key to support long-term policy formation.

In the short term, most of the supranational stakeholders noted that showing compliance with established sustainability criteria and requirements is important. However, the current sustainability criteria for bioenergy are not sufficiently stringent and comprehensive to ensure adequate level of sustainability. Technological and investment risks of further bioenergy deployment have not been fully assessed. Therefore, in tackling these issues, implementation of more efficient markets and great trade flexibilities would help the bioenergy sector grow further.

In the medium term, most supranational stakeholders noted that bioenergy has a role in the renewable energy sector. They stated that the sector needs to focus on the technologies and deployments of advanced fuels. Technological development for processing and flexible measures to mobilise resources should also be further investigated.

Half of the supranational stakeholders were of the view that bioenergy use in heat and electricity will likely not grow significantly in the EU due to feedstock limits and lower conversion efficiency (particularly electricity). However in other regions of abundant biomass resources, heat and electricity generation from biomass would have the potential to grow strongly. Collaboration with other sectors of the bioeconomy needs to be enhanced for long-term development. In addition, sustainability criteria should take local context into account (e.g. FSC and PEFC establish global principles and standards but those principles are applied differently in each country after taking into account geographical, social, economic and environmental conditions).

\section{Assessment of position and level of interests and influence}

This section included own interpretation of the authors on position of the stakeholder groups as well as the level of their interests and influence (Fig. 12). The roundtable dialogues and interviews showed that most Supranational stakeholders have a high awareness and expertise on global issues and a clear vision for bioenergy. Their reflection on the questionnaire results, their vision and their answers to the key questions on bioenergy development also helped the authors to establish a conceptual sketch of the interests and influence of the different stakeholder groups.

The consultation results showed that awareness of the general public is low. Therefore, their interest and influence is assumed to be minor. Environmental NGO groups in average showed a relative interest in bioenergy (when bioenergy is among the larger sectors on which they focus). Other NGOs representing the biomass producers and bioenergy industry were more neutral and supportive of bioenergy. Some environmental NGOs participated in the consultation showed a clear position about bioenergy development with some having concerns about environmental impacts of feedstock sourcing and mobilisation. We perceived that they also have relatively high influence through information and communication campaigns, with a capacity to change position of other groups. Some of them have a critical view towards bioenergy. Certain biomass users for other purposes than bioenergy, such as biochemical and biomaterial stakeholders highlighted concerns over resource competition between bioenergy and their own sectors. However, they indicated a limited influence on bioenergy development.

Biomass producers and biomass users for energy are generally interested in bioenergy and they support bioenergy development. The biomass users for energy are agents working on bioenergy, and it was anticipated that their interest in bioenergy is high. However, their influence is not large, as they are also dependent on policies and long-term governmental strategies and targets for renewable energy. The academia and consulting group showed a high interest in bioenergy, which can also be explained by this being their working field. Given contradicting scientific reports and consultancies on benefits and impacts of the bioenergy sector, their influence on bioenergy development is not directly and not well recognised. Policy-makers have the most important role in designing energy policies which influence the bioenergy development.

\section{Discussion}

To put our results in a wider perspective, we compare them with regional case studies in Canada, Germany and the US of the same project as well as with some previous studies of the same focuses but with a more narrow consultation scope. We find that there are some prevailing points both from a global perspective and at a regional level. The points include sustainability governance, conditions for acceptance and benefits of bioenergy, as well as integration into bioeconomy.

\section{Sustainability governance and certification}

This study confirms the importance of credible sustainability governance and certification at a global level but also at a regional level. Sustainability governance helps to gain and enhance support for bioenergy and thus also for bioenergy development, which was revealed in previous studies as well as in the regional case studies of the same project [12, 13, 17-19]. Results of two regional case studies indicated that certification and standards are also considered effective tools for ensuring sustainability governance $[17,18]$. However, governance of sustainable bioenergy systems is not a major concern when sustainability compliance needs to be demonstrated by 


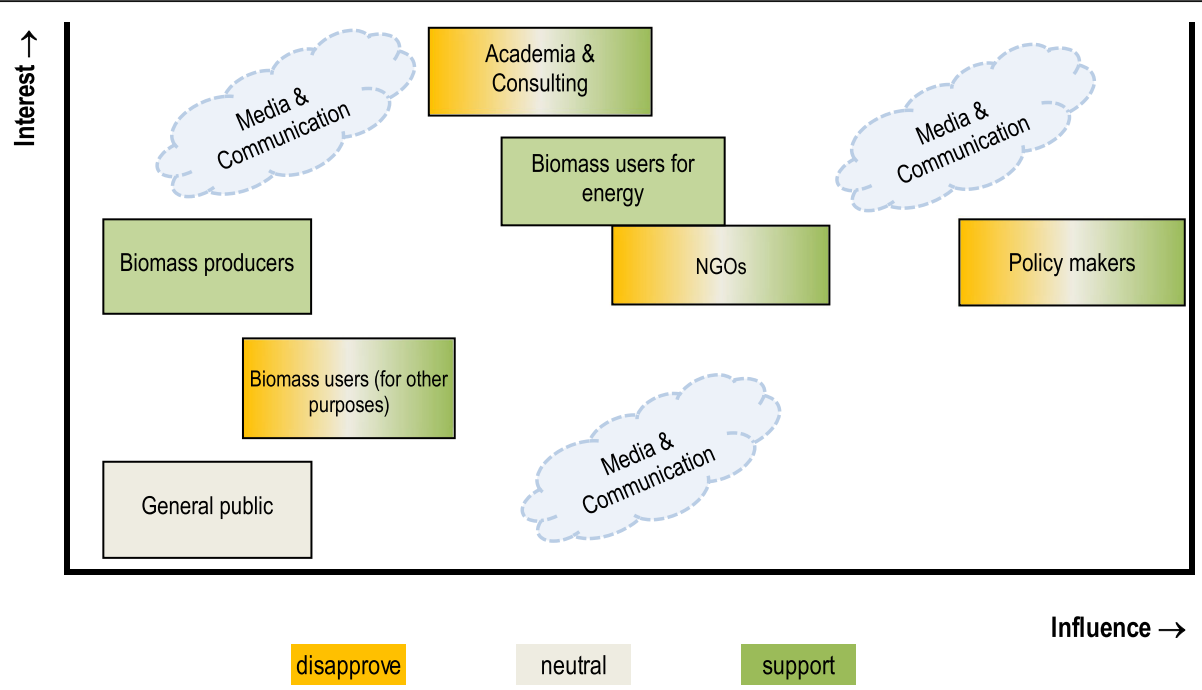

Fig. 12 Conceptual sketch of interest and influence level of stakeholder groups

law or public regulation. Furthermore, transparency and monitoring systems are considered important prerequisites to assure credible sustainability governance [30]. It also shows that a general sustainability framework can be designed at a global level. However, specific sustainability criteria should be tailored for different regions and sustainability measurements and applications need to consider local context.

\section{Acceptance and benefits of bioenergy}

The study results reveal that overall, most participating stakeholder groups hold a relatively positive view of bioenergy; accept the bioenergy sector if sustainability conditions are met and indicate potential benefits of bioenergy if developed in a proper direction. Both the conditions for acceptance and benefits include reduction of environmental impacts, conservation of wildlife and ecosystems, provision of aesthetic benefits, creation of local jobs and contributions to local development. Similarly, at regional level, income creation for individuals and companies as well as a creation of new business opportunities for local communities are found necessary to support bioenergy $[18,19]$.

\section{Integration into bioeconomy}

Bioenergy and other bioeconomy sectors use the same or similar feedstocks and share similar supply chains. Therefore, integrating various bioeconomy sectors should be addressed. The integration helps to avoid resource competition between sectors, recover and reuse resources, while enhancing sectoral collaborations towards a long-term development. This aspect has also been confirmed by previous and regional case studies $[13,17,18]$. One example is the German biogas case study. In Germany, biomass is mobilised to produce biogas which is an integral part in the energy system and used in the chemical industry and as a basis for the worldwide promoted bioeconomy. A rapid capacity growth in the biogas sector combined with a significant increase in meat production and thus fodder production fostered sustainability threats. A sustainable development of biogas therefore needs additional instruments such as a central system regulating the sustainability aspects of biogas apart from the agricultural sector, but also the better implementation of biogas in the further integration into the bioeconomy by going beyond the supply of renewable energies.

\section{Study limitations}

While this study aims to be broad and comprehensive in its scope, it has some limitations. The results were based on data gathered from self-selected respondents, with the number and geographic distribution varying by category; they were not randomly selected from a well-defined population. This means that there are reservations to the interpretation of position and vision as representative for each stakeholder group. New studies may seek to complement the study by engaging more balanced contributions from specific stakeholder groups and from different continents.

\section{Conclusions and recommendations}

This study comprehensively addresses position and vision of various stakeholder groups including the supranational stakeholders, from local to global levels, towards bioenergy. It covers a broad scope of aspects and through the consultation with separate stakeholders groups; their position and vision are drawn more clearly compared to previous results. 


\section{Conclusions}

The study reveals among others that the information and communication channels play important role to frame the position and vision of stakeholders towards bioenergy. The most trusted source of information (academic studies), rather than just media channels, should be strengthened as a basis for developing communication strategies. A continued dialogue and improved communication of scientific evidence of bioenergy's impacts and benefits to external stakeholders could help identify stakeholder priorities and find solutions acceptable for all parties. The potential benefits of bioenergy in its contribution to climate change mitigation, environmental improvements, social and economic enhancements and to the bioeconomy if shown by scientific evidence need to be translated into simple and clear messages to assist long-term decision-making for the bioenergy sector as well as to inform the general public and other stakeholder groups.

Establishment of pathways towards sustainable development and a market-based growth of the bioenergy sector without governmental financial support is decisive to change stakeholders' position and gain more social acceptance of bioenergy and its potential contributions to sustainable development. Options to integrate bioenergy into the bioeconomy and establishment of sustainable supply chains are important for the sector to thrive further in the medium and long term.

To change the stakeholders' position and ultimately to receive or enhance support for the bioenergy sector, sustainability criteria covering social, economic and environmental aspects need to be mandatorily implemented for all types of biomass, regardless of end use, and are as such also applicable to the wider bioeconomy. Sustainability compliance and transparency is likely to be a solution for bioenergy to enhance support in the long term. However, it remains to be seen whether mandatory implementation will ultimately lead to more stakeholder acceptance, and how realistic and rapid implementation for other end uses is. The current certification schemes were not deemed completely credible and transparent by most stakeholder groups.

The sustainability requirements (GHG emissions reduction, SFM, protection of high biodiversity values and carbon stocks, social compliance), which are found in many voluntary schemes, are fundamental but have not met the expectations of all external stakeholders.

The stakeholders indicate the bioenergy sector to consider compliance with additional environmental, social and economic aspects including ecosystem conservation, no competition with food production and no violation of human and land rights. However, these sustainability aspects need to be defined and agreed upon by external stakeholders. Sustainability measures and certification will mean additional costs, adding to the high price of bioenergy relative to fossil alternatives. While these costs may be reduced with increasing uptake of sustainability certification, use of sustainable biomass needs to be also economically profitable and thus may require prolonged policy support.

\section{Recommendations and study limitations}

In addition to the key recommendations shown by the consulted stakeholders, there are certain aspects that the bioenergy sector should also consider. Stakeholders in the bioenergy supply chains are suggested to focus on mobilising what is perceived by stakeholders as sustainable resources, and further advancing of processing technologies, developing more effective supply chains, and ultimately reducing bioenergy costs to aid the sector to grow without subsidies and financial incentives. In addition to currently accepted feedstocks used for bioenergy, stakeholders' position suggests that additional feedstocks from sustainable bioenergy crops and forest biomass can be mobilised. Those feedstocks could potentially be harvested on surplus lands, by afforestation with low iLUC risks, through increased yield per area unit, and increased supply chain integration and efficiency.

We expect that these recommendations, if considered and implemented, will help to change the position of critical external stakeholder groups and ultimately change their vision to positive perspectives of bioenergy development. The findings of the study can be used to inform interested stakeholders on the position of different groups on bioenergy, their awareness and expectations regarding bioenergy development. The findings can also be considered by the bioenergy actors to better communicate the sectoral progresses to external stakeholder groups as well as consider external opinions and recommendations for their long-term development.

\section{Supplementary information}

Supplementary information accompanies this paper at https://doi.org/10. 1186/s13705-019-0225-0.

Additional file 1. Appendix: Online questionnaire.

\section{Abbreviations}

ETS: Emission Trading System; EU: European Union; FAO: Food and Agriculture Organisation; FSC: Forest Stewardship Council; GHG: Greenhouse gases; IEA: International Energy Agency; iLUC: Indirect land use change; ISCC: International Sustainability \& Carbon Certification; IUCN: International Union for Conservation of Nature and Natural Resources; NGO: Not for profit organisation; PEFC: Programme for Endorsement of Forest Certification; RED: Renewable Energy Directive; RSB: The Roundtable on Sustainable Materials; RVO: Rijksdienst voor Ondernemend Nederland; SFM: Sustainable Forest Management; UN: United Nations; WBA: World Bioenergy Association; WWF: World Wide Fund for Nature 


\section{Acknowledgements}

The authors would like to express appreciation to the stakeholders who made contributions to this study. Special thanks go to the Project Advisory Board of the IEA Intertask Sustainability project, Alex Mason (WWF Europe), Ander Evald (HOFOR), Andrew Goldberg (Rainforest Alliance), Ben Larson (Enviva), Carlo Giallombardo (Bioenergy EUROPE), Elena Schmidt (RSB), Fanny-Pomme Langue (Bioenergy EUROPE and Confederation of European Forest Owners), Jori Sihvonen (Transport \& Environment), Klas Sander (World Bank), Maria Michela Morese (Global Bioenergy Partnership), Nathalie Hemeleers (Bioenergy EUROPE), Nora Skjernaa Hansen (Danish Society for Nature Conservation), Olivier Dubois (FAO), Richard Peberdy (Drax), Rolf Hogan (RSB), Sipke Castelein (RVO), and Willem Wiskerke (Greenpeace).

\section{Authors' contributions}

TMM, UF and MJ jointly developed the online survey. TMM analysed and interpreted the online survey data regarding the answers from stakeholder groups. TMM and URF carried out the interviews with the supranational stakeholders while MJ led the roundtable dialogues with invited stakeholders. TMM, MJ and URF assessed the results of the dialogues and interviews. All authors wrote, read and approved the final manuscript.

\section{Funding}

This paper was funded by and written in the frame of the IEA Bioenergy Intertask Sustainability project Measuring, governing and gaining support for sustainable bioenergy supply chains (2016-2018).

\section{Availability of data and materials}

The datasets generated and/or analysed during the current study will soon be available on the website: http://itp-sustainable.ieabioenergy.com/ The datasets used and/or analysed during the current study are available from the corresponding authors on reasonable request.

\section{Ethics approval and consent to participate}

Not applicable

\section{Consent for publication}

Not applicable

\section{Competing interests}

The authors declare that they have no competing interests.

\section{Author details}

'Copernicus Institute of Sustainable Development, University of Utrecht, Vening Meinesz Building A, Princetonlaan 8a, 3508 TA Utrecht, The Netherlands. ${ }^{2}$ International Institute for Sustainability Analysis and Strategy, Heidelberger Str. 129 1/2, 64285 Darmstadt, Germany.

Received: 16 November 2018 Accepted: 25 October 2019

Published online: 19 December 2019

\section{References}

1. IEA. World Energy Outlook 2017. 2017. Available from: https://www.iea.org/ weo2017/. Cited 2018 Sep 9

2. METI (2015) Japan's Energy Plan, p 8 Available from: http://www.enecho. meti.go.jp/en/category/brochures/pdf/energy_plan_2015.pdf. Cited 2018 Aug 7

3. Welfle A (2017) Balancing growing global bioenergy resource demands Brazil's biomass potential and the availability of resource for trade. Biomass Bioenergy 105:83-95

4. MOTTIE (2015) Second Korea Energy Master Plan: Outlook \& Policies To 2035, vol 1

5. Zhao G (2018) Assessment of potential biomass energy production in China towards 2030 and 2050. Int J Sustain Energy 37(1):47-66

6. EC. EU Reference Scenario 2016. 2016. Available from: https://ec.europa.eu/ energy/sites/ener/files/documents/ref2016_report_final-web.pdf. Accessed on 25 Aug 2017

7. IEA (2018) Renewables 2018: Market analysis and forecast from 2018 to 2023, p 211 Available from: https://webstore.iea.org/market-report-seriesrenewables-2018. Cited 2018 Jul 6
8. PricewaterhouseCoopers et al (2017) Sustainable and optimal use of biomass for energy in the EU beyond 2020. VITO, Utrecht University, TU Vienna, INFRO, Rütter Soceco \& PWC

9. EC. Communication on "Clean energy for all Europeans". 2016.

10. IPCC (2018) Global Warming of $1.5^{\circ} \mathrm{C}$ : an IPCC special report on the impacts of global warming of $1.5^{\circ} \mathrm{C}$ above pre-industrial levels and related global greenhouse gas emission pathways, in the context of strengthening the global response to the threat of climate chang, p 36 Available from: http://www.ipcc.ch/report/sr15/. Cited 2018 Oct 10

11. Langeveld JWA (2015) Results of the JRC-SCAR Bioeconomy survey. Scar-Swg-Sbgb.Eu Available from: https://www.scar-swg-sbgb.eu/lw resource/datapool/_items/item_24/survey_bioeconomy_report1501_full_ text.pdf

12. Stupak I, Joudrey J, Smith T, Pelkmans L, Chum H, Cowie A et al (2015) A Global Survey of Stakeholder Views and Experiences for Systems Needed to Effectively and Efficiently Govern Sustainability of Bioenergy. Adv Bioenergy Sustain Chall 5(February):507-534

13. Radics R, Dasmohapatra S, Kelley SS (2015) Systematic review of bioenergy perception studies. BioResources 10(4):8770-8794

14. Peters DM, Wirth K, Böhr B, Ferranti F, Górriz-Mifsud E, Kärkkäinen L et al (2015) Energy wood from forests - stakeholder perceptions in five European countries. Energy Sustain Soc 5(17)

15. Wolsink M (2018) Social acceptance revisited: gaps, questionable trends, and an auspicious perspective. Energy Res Soc Sci 46(May):287-295. https://doi. org/10.1016/j.erss.2018.07.034

16. Mariasiu F (2013) Consumers' Attitudes Related to Biofuel Use in Transportation. Int Rev Manag Mark 3(1):1 Available from: http://search. proquest.com/docview/1283964547? accountid=46437

17. Thrän D et al. Don't hate the player, change the rules: Stakeholder Perceptions in the German Biogas Sector. 2019.

18. Kulisic B, Thiffaut E (2019) Uncharted territories: Expectations towards bioenergy in Canada, a case study in La Tuque (QC)

19. Hodges DG, Chapagain B, Pattarawan W, Poudyal NC, Kline KL, Dale VH (2018) Opportunities and attitudes of private forest landowners in supplying woody biomass for 4 renewable energy. US Dep Energy Available from: http://energy.gov/downloads/doe-public-access-plan

20. Accountability. Aa1000 Stakeholder Engagement Standard 2015. 2015 Available from: http://www.accountability.org/images/content/8/7/875/AA1 OOOSES2015.pdf

21. Röder M (2016) More than food or fuel. Stakeholder perceptions of anaerobic digestion and land use; a case study from the United Kingdom Energy Policy 97(2016):73-81. https://doi.org/10.1016/j.enpol.2016.07.003

22. Reed MS, Graves A, Dandy N, Posthumus H, Hubacek K, Morris J et al (2009) Who's in and why? A typology of stakeholder analysis methods for natural resource management. J Environ Manag 90(5):1933-1949. https://doi.org/1 0.1016/j.jenvman.2009.01.001

23. Environmental NGOs (2016) Proposal to regulate bioenergy production and use in the EU's renewable energy policy framework 2020 - 2030, p 9 Available from: https://www.transportenvironment.org/sites/te/files/ publications/a_new_EU_sustainable_bionenergy_policy_FINAL.pdf. Cited $2017 \operatorname{Jan} 3$

24. RSB (2016) Principles \& Criteria for the Sustainable Production of Biomass, Biofuels and Biomaterials, p 50

25. RVO (2014) Tools for Sustainable Biobased projects, vol 160, Report

26. Greenpeace. Greenpeace bioenergy position paper. 2014

27. Bioenergy Europe. Bioenergy Europe Statistical Report 2018. 2018

28. FAO (2014) FAO Bioenergy \& Food Security Approach: Implementation Guide, $p 25$

29. GBEP (2013) Lessons learned in testing the Global Bio-Energy Partnership sustainability indicators, p 52

30. Junginger M, Fritsche UR, Mai-Moulin T, Thrän D, Thiffaut E, Kline KL, et al. Understanding positions and underlying motivations of stakeholder groups relative to their perceptions of bioenergy. 2019.

\section{Publisher's Note}

Springer Nature remains neutral with regard to jurisdictional claims in published maps and institutional affiliations. 